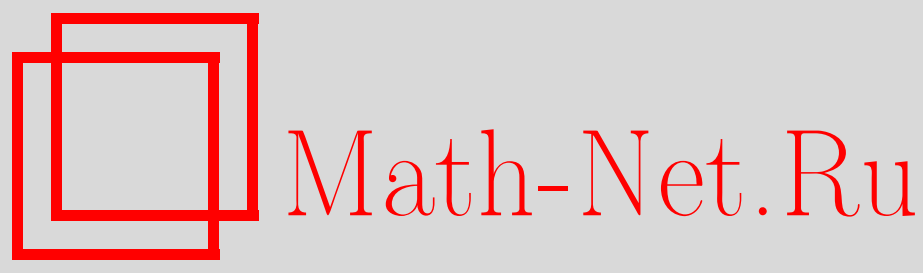

С. Е. Конштейн, И. В. Тютин, Деформации невырожденных постоянных скобки и антискобки Пуассона на суперпространстве произвольной размерности, $Т M \Phi$, 2008, том 155, номер 1, 109-116

DOI: https://doi.org/10.4213/tmf6196

Использование Общероссийского математического портала Math-Net.Ru подразумевает, что вы прочитали и согласны с пользовательским соглашением http://www.mathnet.ru/rus/agreement

Параметры загрузки:

IP : 34.239 .49 .27

26 апреля 2023 г., 12:52:57

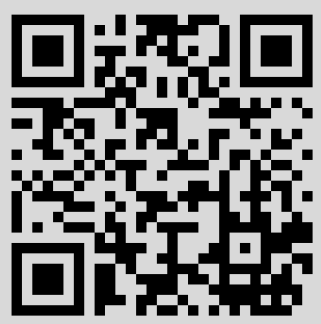




\title{
ДЕФОРМАЦИИ НЕВЫРОЖДЕННЫХ ПОСТОЯННЫХ СКОБКИ И АНТИСКОБКИ ПУАССОНА НА СУПЕРПРОСТРАНСТВЕ ПРОИЗВОЛЬНОЙ РАЗМЕРНОСТИ
}

\begin{abstract}
Рассмотрены (анти)пуассоновские супералгебры с постоянной невырожденной (анти)скобкой, заданной на гладких функциях на $\mathbb{R}^{n}$ с компактным носителем, принимающих значения в алгебре Грассмана. Найдены деформации и центральные расширения таких супералгебр.
\end{abstract}

Ключевые слова: супералгебра Пуассона, антипуассоновская супералгебра, деформация суперскобок, когомологии супералгебры, квантование.

\section{1. ВВЕДЕНИЕ}

Надежда построить квантовую механику на нетривиальных многообразиях связана с геометрическим, или деформационным, квантованием [1]-[4]. С операторами ассоциируются функции на фазовом пространстве, а произведение и коммутатор операторов описываются ассоциативным *-произведением и $*$-коммутатором функций. Эти *-произведение и *-коммутатор являются деформациями обычного произведения и обычной скобки Пуассона. Имеется много публикаций, посвященных деформациям (анти)скобки Пуассона для различных функциональных пространств. В работе [5] эта проблема рассмотрена для супералгебры Пуассона, реализованной на суперпространстве полиномов, и для антискобки. Чисто грассманов случай рассмотрен в [6]. Бозонная алгебра Пуассона, реализованная на гладких функциях, рассматривалась в [7].

Результат зависит от выбора функционального пространства (подробнее см. [5]).

В данной статье изложены результаты работ [8]-[11], где был найден общий вид *-коммутатора для случая пуассоновской и антипуассоновской супералгебр, реализованных на гладких функциях с компактным носителем, принимающих значения в алгебре Грассмана, а также для случая центральных расширений указанных супералгебр.

*Физический институт им. П. Н. Лебедева РАН, Москва, Россия. E-mail: konstein@lpi.ru, tyutin@lpi.ru 
1.1. Деформации топологических супералгебр Ли. Напомним некоторые понятия, связанные с формальными деформациями алгебр (см., например, [12]), применительно к случаю топологических супералгебр Ли. Пусть $L$ - топологическая супералгебра Ли над $\mathbb{K}(\mathbb{K}=\mathbb{R}$ или $\mathbb{C})$ с суперскобкой Ли $\{\cdot, \cdot\}, \mathbb{K}\left[\left[\hbar^{2}\right]\right]$ - кольцо формальных степеннь́х рядов по $\hbar^{2}$ над $\mathbb{K}$. Обозначим $\mathbb{K}\left[\left[\hbar^{2}\right]\right]$-модуль формальных степеннь́х рядов по $\hbar^{2}$ с коэффициентами в $L$ через $L\left[\left[\hbar^{2}\right]\right]$ и снабдим $\mathbb{K}\left[\left[\hbar^{2}\right]\right]$ и $L\left[\left[\hbar^{2}\right]\right]$ топологией прямого произведения. Градуировка в $L$ естественно определяет градуировку в $L\left[\left[\hbar^{2}\right]\right]$ : элемент $f=f_{0}+\hbar^{2} f_{1}+\cdots$ имеет определенную четность $\varepsilon(f)$, если $\varepsilon(f)=\varepsilon\left(f_{j}\right)$ для всех $j=0,1, \ldots$ Всякое $p$-линейное раздельно-непрерывное отображение из $L^{p}$ в $L$ (в частности, скобка $\left.\{\cdot, \cdot\}\right)$ единственным образом продолжается вследствие $\mathbb{K}\left[\left[\hbar^{2}\right]\right]$-линейности до $p$-линейного раздельно-непрерывного отображения над $\mathbb{K}\left[\left[\hbar^{2}\right]\right]$ из $L\left[\left[\hbar^{2}\right]\right]^{p}$ в $L\left[\left[\hbar^{2}\right]\right]$. По определению (непрерывная) формальная деформация $L$ - это $\mathbb{K}\left[\left[\hbar^{2}\right]\right]$-билинейная непрерывная по каждому аргументу в отдельности суперскобка Ли $C(\cdot, \cdot)$ на $L\left[\left[\hbar^{2}\right]\right]$ такая, что $C(f, g)=\{f, g\} \bmod \hbar^{2}$ для любых $f, g \in L\left[\left[\hbar^{2}\right]\right]$. Очевидно, что всякая формальная деформация $C$ представима в виде

$$
C(f, g)=\{f, g\}+\hbar^{2} C_{1}(f, g)+\hbar^{4} C_{2}(f, g)+\cdots, \quad f, g \in L,
$$

где $C_{j}$ - раздельно-непрерывные суперкососимметричные билинейные отображения из $L \times L$ в $L$ (2-коцепи с коэффициентами в присоединенном представлении $L)$. Формальные деформации $C^{1}$ и $C^{2}$ называются эквивалентными, если существует такой непрерывный $\mathbb{K}\left[\left[\hbar^{2}\right]\right]$-линейный оператор $T: L\left[\left[\hbar^{2}\right]\right] \rightarrow L\left[\left[\hbar^{2}\right]\right]$, что $T C^{1}(f, g)=$ $C^{2}(T f, T g), f, g \in L\left[\left[\hbar^{2}\right]\right]$ и $T=\mathrm{id}+\hbar^{2} T_{1}$. Задача поиска формальных деформаций $L$ тесно связана с задачей вычисления когомологий Шевалле-Эйленберга супералгебры $L$ с коэффициентами в присоединенном представлении $L$. Пусть $\mathcal{C}_{p}(L)$ обозначает пространство $p$-линейных суперкососимметричных раздельно-непрерывных отображений из $L^{p}$ в $L$ (пространство $p$-коцепей с коэффициентами в присоединенном представлении $L$ ). Пространство $\mathcal{C}_{p}(L)$ имеет естественную $\mathbb{Z}_{2}$-градуировку: по определению $M_{p} \in \mathcal{C}_{p}(L)$ имеет определенную четность $\varepsilon\left(M_{p}\right)$, если соотношение

$$
\varepsilon\left(M_{p}\left(f_{1}, \ldots, f_{p}\right)\right)=\varepsilon\left(M_{p}\right)+\varepsilon\left(f_{1}\right)+\cdots+\varepsilon\left(f_{p}\right)
$$

справедливо при любых $f_{j} \in L$ с определенными четностями $\varepsilon\left(f_{j}\right)$. Поскольку суперскобка Ли всегда четная, все $C_{j}$ в разложении (1) должны быть четными 2 -коцепями. Дифференциал $d_{p}^{\text {ad }}$ определяется [13] как такой линейный оператор из $\mathcal{C}_{p}(L)$ в $\mathcal{C}_{p+1}(L)$, что

$$
\begin{aligned}
d_{p}^{\mathrm{ad}} M_{p}\left(f_{1}, \ldots, f_{p+1}\right)= \\
=-\sum_{j=1}^{p+1}(-1)^{j+\varepsilon\left(f_{j}\right)|\varepsilon(f)|_{1, j-1}+\varepsilon\left(f_{j}\right) \varepsilon_{M_{p}}}\left\{f_{j}, M_{p}\left(f_{1}, \ldots, \hat{f}_{j}, \ldots, f_{p+1}\right)\right\}- \\
\quad-\sum_{i<j}(-1)^{j+\varepsilon\left(f_{j}\right)|\varepsilon(f)|_{i+1, j-1}} M_{p}\left(f_{1}, \ldots, f_{i-1},\left\{f_{i}, f_{j}\right\}, f_{i+1}, \ldots, \hat{f}_{j}, \ldots, f_{p+1}\right)
\end{aligned}
$$

для любых $M_{p} \in \mathcal{C}_{p}(L)$ и $f_{1}, \ldots, f_{p+1} \in L$, имеющих определенные четности. Здесь

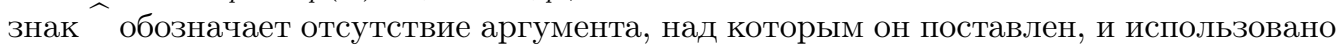
обозначение $|\varepsilon(f)|_{i, j}=\sum_{l=i}^{j} \varepsilon\left(f_{l}\right)$. Записывая тождество Якоби для деформации $C$ 
вида (1),

$$
(-1)^{\varepsilon(f) \varepsilon(h)} C(f, C(g, h))+\operatorname{cycle}(f, g, h)=0,
$$

и рассматривая члены порядка $\hbar^{2}$, мы находим, что $d_{2}^{\text {ad }} C_{1}=0$. Таким образом, деформации первого порядка супералгебры $L$ описываются 2-коциклами дифференциала $d^{\mathrm{ad}}$.

1.2. Супералгебра Пуассона. Обозначим через $\mathcal{D}\left(\mathbb{R}^{k}\right)$ пространство гладких $\mathbb{K}$-значных функций на $\mathbb{R}^{k}$ с компактным носителем, снабженное своей стандартной топологией: последовательность $\varphi_{k} \in \mathcal{D}\left(\mathbb{R}^{k}\right)$ сходится к $\varphi \in \mathcal{D}\left(\mathbb{R}^{k}\right)$, если $\partial^{\lambda} \varphi_{k}$ сходится равномерно к $\partial^{\lambda} \varphi$ для любого мультииндекса $\lambda$ и носители всех $\varphi_{k}$ содержатся в некотором компактном множестве. Положим

$$
D_{n_{+}}^{n_{-}}=\mathcal{D}\left(\mathbb{R}^{n_{+}}\right) \otimes \mathbb{G}^{n_{-}}, \quad E_{n_{+}}^{n_{-}}=C^{\infty}\left(\mathbb{R}^{n_{+}}\right) \otimes \mathbb{G}^{n_{-}},
$$

где $\mathbb{G}^{n_{-}}$- алгебра Грассмана с $n_{-}$образующими. Образующие алгебры Грассмана (соответственно координаты пространства $\mathbb{R}^{n_{+}}$) мы обозначаем через $\xi_{\alpha}, \alpha=$ $1,2, \ldots, n_{-}$(соответственно $\left.x_{i}, i=1,2, \ldots, n_{+}\right)$. Мы будем также использовать коллективные переменные $z_{A}$, которые равны $x_{A}$ при $A=1,2, \ldots, n_{+}$и равны $\xi_{A-n_{+}}$ при $A=n_{+}+1, \ldots, n_{+}+n_{-}$. Пространства $D_{n_{+}}^{n_{-}}$и $E_{n_{+}}^{n_{-}}$обладают естественной градуировкой, определяемой градуировкой алгебры Грассмана. Четность элемента $f$ этих пространств обозначается через $\varepsilon(f)$. Положим также $\varepsilon_{A}=0$ для $A=1,2, \ldots, n_{+}$и $\varepsilon_{A}=1$ для $A=n_{+}+1, \ldots, n_{+}+n_{-}$.

Пусть $\partial / \partial z_{A}$ и $\overleftarrow{\partial} / \partial z_{A}-$ операторы левого и правого дифференцирования. Скобка Пуассона определяется соотношением

$$
\{f, g\}(z)=\sum_{A, B} f(z) \frac{\overleftarrow{\partial}}{\partial z_{A}} \omega^{A B} \frac{\partial}{\partial z_{B}} g(z)=-(-1)^{\varepsilon(f) \varepsilon(g)}\{g, f\}(z)
$$

где симплектическая метрика $\omega^{A B}=-(-1)^{\varepsilon_{A} \varepsilon_{B}} \omega^{B A}$ является постоянной невырожденной матрицей. Для определенности выберем ее в виде

$$
\omega^{A B}=\left(\begin{array}{cc}
\omega^{i j} & 0 \\
0 & \lambda_{\alpha} \delta^{\alpha \beta}
\end{array}\right), \quad \lambda_{\alpha}= \pm 1, \quad i, j=1,2, \ldots, n_{+}, \quad \alpha, \beta=1,2, \ldots, n_{-},
$$

где $\omega^{i j}$ - каноническая симплектическая форма (если $\mathbb{K}=\mathbb{C}$, то можно выбрать $\left.\lambda_{\alpha}=1\right)$. Невырожденность матрицы $\omega^{A B}$ означает, в частности, что $n_{+}$четно. Скобка Пуассона удовлетворяет тождеству Якоби

$$
(-1)^{\varepsilon(f) \varepsilon(h)}\{f,\{g, h\}\}(z)+\operatorname{cycle}(f, g, h)=0, \quad f, g, h \in E_{n_{+}}^{n_{-}} .
$$

Супералгеброй Пуассона $\mathcal{P}_{n_{+}}^{n_{-}}$мы называем пространство $D_{n_{+}}^{n_{-}}$со скобкой Пуассона (2) на нем. Соотношения (2) и (3) показывают, что эта скобка действительно определяет структуру супералгебры Ли на $D_{n_{+}}^{n_{-}}$.

Интеграл на $D_{n_{+}}^{n_{-}}$определяется соотношением

$$
\bar{f} \stackrel{\text { def }}{=} \int d z f(z)=\int_{\mathbb{R}^{n_{+}}} d x \int d \xi f(z)
$$

где интеграл по алгебре Грассмана нормирован условием $\int d \xi \xi_{1} \ldots \xi_{n_{-}}=1$.

Введем супералгебру $Z_{n_{+}}^{n_{-}}, D_{n_{+}}^{n_{-}} \subset Z_{n_{+}}^{n_{-}} \subset E_{n_{+}}^{n_{-}}, Z_{n_{+}}^{n_{-}}=D_{n_{+}}^{n_{-}} \oplus \mathcal{C}_{D_{n_{+}}^{n_{-}}}\left(E_{n_{+}}^{n_{-}}\right)$, где $\mathcal{C}_{D_{n_{+}}^{n_{-}}}\left(E_{n_{+}}^{n_{-}}\right)$- централизатор $D_{n_{+}}^{n_{-}}$в $E_{n_{+}}^{n_{-}}$. 


\section{2. ДЕФОРМАЦИИ СУПЕРАЛГЕБРЫ ПУАССОНА}

Для любого $\varkappa \in \mathbb{K}[[\hbar]]$ такого, что $c_{1} \stackrel{\text { def }}{=} \hbar^{2} \varkappa^{2} / 6 \in \hbar^{2} \mathbb{K}\left[\left[\hbar^{2}\right]\right]$, суперскобка Мойала

$$
\mathcal{M}_{c_{1}}(z \mid f, g)=\frac{1}{\hbar \varkappa} f(z) \operatorname{sh}\left(\hbar \varkappa \sum_{A, B} \frac{\overleftarrow{\partial}}{\partial z_{A}} \omega^{A B} \frac{\partial}{\partial z_{B}}\right) g(z)
$$

кососимметрична и удовлетворяет тождеству Якоби. Следовательно, она является деформацией исходной алгебры Пуассона.

Определим билинейные отображения $m_{3}$ и $m_{\zeta}$ из $\left(D_{n_{+}}^{n_{-}}\right)^{2}$ в $D_{n_{+}}^{n_{-}}$соотношениями

$$
\begin{aligned}
& m_{3}(z \mid f, g)=\mathcal{E}_{z} f(z) \bar{g}-(-1)^{\varepsilon(f) \varepsilon(g)} \mathcal{E}_{z} g(z) \bar{f}, \\
& m_{\zeta}(z \mid f, g)=\{\zeta(z), f(z)\} \bar{g}-(-1)^{\varepsilon(f) \varepsilon(g)}\{\zeta(z), g(z)\} \bar{f},
\end{aligned}
$$

где $\mathcal{E}_{z} \stackrel{\text { def }}{=} 1-z \partial_{z} / 2, z=\left(x_{1}, \ldots, x_{n}, \xi_{1}, \ldots, \xi_{m}\right)$.

Для $\zeta \in E_{n_{+}}^{n_{-}}\left[\left[\hbar^{2}\right]\right]$ и $c_{1}, c \in \mathbb{K}\left[\left[\hbar^{2}\right]\right]$ положим

$$
\begin{aligned}
C_{\zeta, c_{1}}^{(1)}(z \mid f, g) & =\mathcal{M}_{c_{1}}(z \mid f+\zeta \bar{f}, g+\zeta \bar{g}), \\
C_{\zeta, c_{1}, c}^{(1)}(z \mid f, g) & =\mathcal{M}_{c_{1}}(z \mid f+\zeta \bar{f}, g+\zeta \bar{g})+c \bar{f} \bar{g} .
\end{aligned}
$$

\section{1. Деформации супералгебры Пуассона при $\left(n_{+} \mid n_{-}\right) \neq(2 \mid 2)$.}

Теорема 1 [8], [9]. 1. Пустъ $n_{-}=2 k,\left(n_{+} \mid n_{-}\right) \neq(2 \mid 2)$. Тогда всякая непрерывная формальная деформачия супералгебры Пуассона $\mathcal{P}_{n_{+}}^{n_{-}}$эквивалентна либо суперскобке $C_{\zeta, c_{1}}^{(1)}(z \mid f, g)$ при некоторой четной функции $\zeta \in \hbar^{2} E_{n_{+}}^{n_{-}}\left[\left[\hbar^{2}\right]\right] u$ $c_{1} \in \hbar^{2} \mathbb{K}\left[\left[\hbar^{2}\right]\right]$, либо суперскобке

$$
C_{\zeta, c_{3}}^{(3)}(z \mid f, g)=\{f(z), g(z)\}+m_{\zeta}(z \mid f, g)+c_{3} m_{3}(z \mid f, g)
$$

при некоторой четной функиии $\zeta \in \hbar^{2} E_{n_{+}}^{n_{-}}\left[\left[\hbar^{2}\right]\right]$ u $c_{3} \in \hbar^{2} \mathbb{K}\left[\left[\hbar^{2}\right]\right]$. Деформаиии $C_{\zeta_{1}, c}^{(i)}$ и $C_{\zeta_{2}, c}^{(i)}$ эквивалентны, если $\zeta_{1}-\zeta_{2} \in Z_{n_{+}}^{n_{-}}\left[\left[\hbar^{2}\right]\right]$.

2. Пусть $n_{-}=2 k+1$. Тогда всякая непрерывная формальная деформация супералгебры Пуассона $\mathcal{P}_{n_{+}}^{n_{-}}$эквивалентна суперскобке $C_{\zeta, c_{1}, c}^{(1)}(z \mid f, g)$ при некоторых $c, c_{1} \in \hbar^{2} \mathbb{K}\left[\left[\hbar^{2}\right]\right]$ и нечетной функиии $\zeta \in \hbar^{2} E_{n_{+}}^{n_{-}}\left[\left[\hbar^{2}\right]\right] \operatorname{ma\kappa их,~что~}\left[\mathcal{M}_{c_{1}}(z \mid \zeta, \zeta)+c\right] \in$ $D_{n_{+}}^{n_{-}}\left[\left[\hbar^{2}\right]\right]$. Деформачии $C_{\zeta_{1}, c_{1}, c}^{(1)} u C_{\zeta_{2}, c_{1}, c}^{(1)}$ эквивалентны, если $\zeta_{1}-\zeta_{2} \in Z_{n_{+}}^{n_{-}}\left[\left[\hbar^{2}\right]\right]$.

2.2. Деформации супералгебры Пуассона при $\left(n_{+} \mid n_{-}\right)=(2 \mid 2)$.

ТЕорема 2 [9]. Пусть $n_{+}=n_{-}=2$ и функиия $\Lambda \in C^{\infty}(\mathbb{R})$ такова, что $d \Lambda / d x$ имеет компактный носитель, $\Lambda(-\infty)=0 u \Lambda(+\infty)=1$. Положим

$$
\begin{aligned}
& \Delta(x \mid f) \stackrel{\text { def }}{=} \int d \xi f(x, \xi), \\
& \Theta(x \mid f) \stackrel{\text { def }}{=} \int d u \delta\left(x_{1}-u_{1}\right) \theta\left(x_{2}-u_{2}\right) f(u), \\
& \Psi(x \mid f) \stackrel{\text { def }}{=} \Lambda\left(x_{2}\right) \int d^{2} y d \eta \theta\left(x_{1}-y_{1}\right) f(y, \eta), \\
& \Xi(x \mid f) \stackrel{\text { def }}{=} \Theta(x \mid f)-\Lambda\left(x_{2}\right) \int d u \delta\left(x_{1}-u_{1}\right) f(u),
\end{aligned}
$$


где $z=\left(x_{1}, x_{2}, \xi_{1}, \xi_{2}\right), u=\left(y_{1}, y_{2}, \eta_{1}, \eta_{2}\right)$.

Пусть билинейные отображения $m_{3} u m_{\zeta}$ определень формулами (4) u (5). Определим билинейное отображение $m_{5}$ из $\left(D_{2}^{2}\right)^{2}$ в $D_{2}^{2}$ соотношениями

$$
\begin{aligned}
m_{5}(z \mid f, g) & =m_{51}(z \mid f, g)+m_{52}(z \mid f, g)+m_{53}(z \mid f, g)+m_{54}(z \mid f, g), \\
m_{51}(z \mid f, g) & =f(z) \Delta(x \mid g)-\Delta(x \mid f) g(z), \\
m_{52}(z \mid f, g) & =2 \Theta(x \mid f) \partial_{2} g(z)-2 \partial_{2} f(z) \Theta(x \mid g), \\
m_{53}(z \mid f, g) & =-2\{f(z), \Psi(x \mid g)\}+2\{g(z), \Psi(x \mid f)\}, \\
m_{54}(z \mid f, g) & =\Xi\left(x \mid \partial_{2} f g-f \partial_{2} g\right) .
\end{aligned}
$$

Определим билинейные формы $\sigma_{3}, \sigma_{\zeta} u B_{\zeta}$ :

$$
\begin{aligned}
\sigma_{3}(f, g)= & 4 \widetilde{\Theta}(g)\left(\Xi(f) \partial_{2} \Lambda-\Xi\left(f \partial_{2} \Lambda\right)\right)-4 \widetilde{\Theta}(f)\left(\Xi(g) \partial_{2} \Lambda-\Xi\left(g \partial_{2} \Lambda\right)\right), \\
\sigma_{\zeta}(f, g)= & A_{\zeta}(f) \bar{g}-A_{\zeta}(g) \bar{f}, \\
A_{\zeta}(f)= & f \Delta(\zeta)-\Delta(f) \zeta+\Xi\left(\partial_{2} f \zeta-f \partial_{2} \zeta\right)+2 \partial_{1} \zeta \partial_{2} \Lambda \widetilde{\Theta}(f)+ \\
& \quad+2 \partial_{2} \zeta \Lambda \Xi(f)-2 \partial_{2} f \int \theta\left(x_{2}-y_{2}\right) \theta\left(y_{2}\right) \delta\left(x_{1}-y_{1}\right) \zeta(u) d u, \\
& B_{\zeta}(f, g)=4(\widetilde{\Theta}(f) \bar{g}-\widetilde{\Theta}(g) \tilde{f})\left[\Xi\left(\zeta \partial_{2} \Lambda\right)-\partial_{2} \Lambda \int \theta\left(x_{2}-y_{2}\right) \theta\left(y_{2}\right) \delta\left(x_{1}-y_{1}\right) \zeta(u) d u\right] .
\end{aligned}
$$

Тогда всякая непрерывная формальная деформачия супералгебры Пуассона $\mathcal{P}_{2}^{2}$ эквивалентна одной из следующих суперскобок: $C_{\zeta, c_{1}}^{(1)}(z \mid f, g), C_{\zeta, c_{3}}^{(3)}(z \mid f, g)(6)$,

$$
\begin{aligned}
C_{\zeta, c_{5}}^{(5)}(z \mid f, g)=\{ & f(z), g(z)\}+m_{\zeta}(z \mid f, g)+c_{5}\left[m_{5}(z \mid f, g)-\sigma_{\zeta}(z \mid f, g)\right]+ \\
& +c_{5}^{2}\left[B_{\zeta}(z \mid f, g)-\sigma_{3}(z \mid f, g)\right],
\end{aligned}
$$

где $c_{1}, c_{3}, c_{5} \in \hbar^{2} \mathbb{K}\left[\left[\hbar^{2}\right]\right]$ и $\zeta \in \hbar^{2} E_{2}^{2}\left[\left[\hbar^{2}\right]\right]$ - четная функиия.

Деформачии $C_{\zeta_{1}, c}^{(i)}$ и $C_{\zeta_{2}, c}^{(i)}$ эквивалентнь, если $\zeta_{1}-\zeta_{2} \in Z_{n_{+}}^{n_{-}}\left[\left[\hbar^{2}\right]\right]$.

\section{3. ЦЕНТРАЛЬНОЕ РАСШИРЕНИЕ}

Пусть $\mathbf{L}$ - центральное расширение супералгебры $L$, т.е. $\mathbf{L}=L \oplus c, \mathbf{f}=f+a \mathcal{J} \in \mathbf{L}$, $a \mathcal{J} \in c, \varepsilon(\mathcal{J})=0, a \in \mathbb{K}$. Скобку на $\mathbf{L}$ обозначим через $[\mathbf{f}, \mathbf{g}]=-(-1)^{\varepsilon(\mathbf{f}) \varepsilon(\mathbf{g})}[\mathbf{g}, \mathbf{f}]$,

$$
[f, g]=\{f, g\}+Q(f, g) \mathcal{J}, \quad Q(f, g) \in \mathbb{K}, \quad[\mathbf{f}, \mathcal{J}]=0 .
$$

Тогда $Q(f, g)=-(-1)^{\varepsilon(f) \varepsilon(g)} Q(g, f), \varepsilon(Q)=0$, и $Q$ порождает вторую когомологию в тривиальном представлении алгебры $L$ :

$$
d_{2}^{\operatorname{tr}} Q(f, g, h) \stackrel{\text { def }}{=} Q(\{f, g\}, h)-(-1)^{\varepsilon(g) \varepsilon(h)} Q(\{f, h\}, g)-Q(f,\{g, h\})=0 .
$$

Теорема 3 [14]. Определим билинейные формы $Q_{2}^{1}$ и $Q_{2}^{2}$ соотношениями

$$
\begin{gathered}
Q_{2}^{1}(f, g)=\bar{f} \bar{g}, \\
Q_{2}^{2}(f, g)=\int d z\left(f(z) \mathcal{E}_{z} g(z)-(-1)^{\varepsilon(f) \varepsilon(g)} g(z) \mathcal{E}_{z} f(z)\right), \quad f, g \in D_{n_{+}}^{n_{-}},
\end{gathered}
$$

где

$$
\mathcal{E}_{z} \stackrel{\text { def }}{=} 1-\frac{1}{2} z^{A} \frac{\partial}{\partial z^{A}}
$$


Если $n_{-}$четно и $n_{-} \neq n_{+}+4$, то $H_{\mathrm{tr}}^{2}=0 ;$ если $n_{-}=n_{+}+4$, то $H_{\mathrm{tr}}^{2} \simeq \mathbb{K} u$ форма $Q_{2}^{2}$ является нетривиальным кочиклом; если $n_{-}$нечетно, то $H_{\mathrm{tr}}^{2} \simeq \mathbb{K} u$ форма $Q_{2}^{1}$ является нетривиальным коц,иклом.

Из теоремы 3 следует, что у супералгебры Пуассона $D_{n_{+}}^{n_{-}}$имеется центральное расширение $\mathbf{D}_{n_{+}}^{n_{-}}$либо при нечетном $n_{-}$, либо при $n_{-}=n_{+}+4$.

\section{1. Случай $n_{-}=2 k+1$.}

Teорема 4 [10]. Пусть $n_{-}=2 k+1$, где $k$ - целое число, функиии $\zeta(z), w(z) \in$ $\hbar^{2} E_{n_{+}}^{2 k+1}\left[\left[\hbar^{2}\right]\right]$ при некотором с 1 удовлетворяют условиям

$$
\begin{aligned}
& \varepsilon(\zeta)=1, \quad \varepsilon(w)=0, \\
& w(z)=-\mathcal{M}_{c_{1}}(z \mid \zeta, \zeta) .
\end{aligned}
$$

Пустъ $\mathbf{N}_{\zeta, c_{1}}(\mathbf{f}, \mathbf{g})=N_{\zeta, c_{1}}(z \mid \mathbf{f}, \mathbf{g})+n_{\zeta, c_{1}}(\mathbf{f}, \mathbf{g}) \mathcal{J}$, , де

$$
\begin{gathered}
N_{\zeta, c_{1}}(z \mid f, g)=\mathcal{M}_{c_{1}}(z \mid f+\zeta \bar{f}, g+\zeta \bar{g})+w(z) \bar{f} \bar{g} \\
N_{\zeta, c_{1}}(z \mid f, \mathcal{J})=\mathcal{M}_{c_{1}}\left(z \mid w, f+\hbar^{2} \zeta \bar{f}\right), \\
n_{\zeta, c_{1}}(f, g)=\bar{f} \bar{g}, \quad n_{\zeta, c_{1}}(f, \mathcal{J})=0, \quad \mathbf{N}_{\zeta, c_{1}}(z \mid \mathcal{J}, \mathcal{J})=0 .
\end{gathered}
$$

Тогда:

1) каждая непрерывная формальная деформация супералгебры $\mathbf{D}_{n_{+}}^{2 k+1}$ эквивалентна суперскобке $[\mathbf{f}, \mathbf{g}]_{\zeta, c_{1}}=\mathbf{N}_{\zeta, c_{1}}(\mathbf{f}, \mathbf{g})$ при некоторых $c_{1} \in \hbar^{2} \mathbb{K}\left[\left[\hbar^{2}\right]\right]$ и $\zeta(z) \in$ $\hbar^{2} E_{n_{+}}^{2 k+1}\left[\left[\hbar^{2}\right]\right]$, удовлетворяющих (7), (8);

2) суперскобка $[\mathbf{f}, \mathbf{g}]_{\zeta_{1}, c_{1}}$ эквивалентна суперскобке $[\mathbf{f}, \mathbf{g}]_{\zeta_{2}, c_{1}}$, если $\zeta_{1}-\zeta_{2} \in$ $Z_{n_{+}}^{n_{-}}\left[\left[\hbar^{2}\right]\right]$.

3.2. Случай $n_{-}=n_{+}+4$.

TeOPEMA 5 [10]. Пусть $n_{-}=n_{+}+4, \zeta(z) \in \hbar^{2} E_{n_{+}}^{n_{+}+4}\left[\left[\hbar^{2}\right]\right], c_{3} \in \hbar^{2} \mathbb{K}\left[\left[\hbar^{2}\right]\right]$,

$$
\begin{aligned}
Q(f, g)= & \int d z\left(f(z) \mathcal{E}_{z} g(z)-(-1)^{\varepsilon(f) \varepsilon(g)} g(z) \mathcal{E}_{z} f(z)\right), \\
\mathbf{S}_{\zeta, c_{3}}(\mathbf{f}, \mathbf{g})= & S_{\zeta, c_{3}}(z \mid \mathbf{f}, \mathbf{g})+s_{\zeta, c_{3}}(\mathbf{f}, \mathbf{g}) \mathcal{J}, \\
S_{\zeta, c_{3}}(z \mid f, g)=\{ & f(z), g(z)\}+c_{3}\left(\bar{f} \mathcal{E}_{z} g(z)-(-1)^{\varepsilon(f) \varepsilon(g)} \bar{g} \mathcal{E}_{z} f(z)\right)+ \\
& \quad+\left(\{\zeta(z), g(z)\} \bar{f}-(-1)^{\varepsilon(f) \varepsilon(g)}\{\zeta(z), f(z)\} \bar{g}\right), \\
s_{\zeta, c_{3}}(f, g)= & Q(f, g)+Q(\zeta, g) \bar{f}-(-1)^{\varepsilon(f) \varepsilon(g)} Q(\zeta, f) \bar{g}, \\
S_{\zeta, c_{3}}(\mathbf{f}, \mathcal{J})= & s_{\zeta, c_{3}}(\mathbf{f}, \mathcal{J})=0 .
\end{aligned}
$$

Тогда:

1) каждая непрерывная формальная деформация супералгебры $\mathbf{D}_{n_{+}}^{n_{+}+4}$ эквивалентна суперскобке $[\mathbf{f}, \mathbf{g}]_{\zeta, c_{3}}=\mathbf{S}_{\zeta, c_{3}}(\mathbf{f}, \mathbf{g})$ при некоторых $c_{3} \in \hbar^{2} \mathbb{K}\left[\left[\hbar^{2}\right]\right]$ и $\zeta(z) \in$ $\hbar^{2} E_{n_{+}}^{n_{+}+4}\left[\left[\hbar^{2}\right]\right]$;

2) суперскобка $[\mathbf{f}, \mathbf{g}]_{\zeta_{1}, c_{3}}$ эквивалентна суперскобке $[\mathbf{f}, \mathbf{g}]_{\zeta, c_{3}}$, если $\zeta_{1}(z)-\zeta(z) \in$ $Z_{n_{+}}^{n_{-}}\left[\left[\hbar^{2}\right]\right]$. 


\section{4. АНТИСКОБКА}

На пространствах $D_{n_{+}}^{n_{-}}$и $E_{n_{+}}^{n_{-}}$имеется еще одна $\mathbb{Z}_{2}$-градуировка $\epsilon(\epsilon$-четность $)$, обратная $\varepsilon$-четности: $\epsilon=\varepsilon+1$.

Положим $\varepsilon_{A}=0, \epsilon_{A}=1$ при $A=1,2, \ldots, n_{+}$и $\varepsilon_{A}=1, \epsilon_{A}=0$ при $A=$ $n_{+}+1, \ldots, n_{+}+n_{-}$.

Хорошо известно, что при $n_{+}=n_{-}=n$ скобка

$$
[f, g](z)=\sum_{i=1}^{n}\left(f(z) \frac{\overleftarrow{\partial}}{\partial x_{i}} \frac{\partial}{\partial \xi_{i}} g(z)-f(z) \frac{\overleftarrow{\partial}}{\partial \xi_{i}} \frac{\partial}{\partial x_{i}} g(z)\right)
$$

которую мы, следуя [15], называем антискобкой или нечетной скобкой, задает структуру супералгебры Ли на суперпространствах $D_{n} \stackrel{\text { def }}{=} D_{n}^{n}$ и $E_{n} \stackrel{\text { def }}{=} E_{n}^{n}$ с $\epsilon$-четностью.

В самом деле, $[f, g]=-(-1)^{\epsilon(f) \epsilon(g)}[g, f], \epsilon([f, g])=\epsilon(f)+\epsilon(g)$ и выполнено тождество Якоби

$$
(-1)^{\epsilon(f) \epsilon(h)}[f,[g, h]]+(-1)^{\epsilon(g) \epsilon(f)}[g,[h, f]]+(-1)^{\epsilon(h) \epsilon(g)}[h,[f, g]]=0, \quad f, g, h \in E_{n} .
$$

Мы будем называть эти супералгебры Ли антипуассоновскими супералгебрами.

Нечетная скобка Пуассона играет важную роль в лагранжевой формулировке квантовой теории калибровочных полей, известной как БВ-формализм [15], [16] (см. также [17]-[19], [5]). В физической литературе нечетная скобка была введена в [15]. По своим свойствам антискобка во многом аналогична четной скобке Пуассона и даже может быть получена при помощи "канонического формализма" с нечетным временем. Однако в отличие от случая четной скобки Пуассона, для которого имеется обширная литература, посвященная различным аспектам деформации (квантования) алгебры Пуассона, проблема деформации антискобки до сих пор недостаточно изучена.

Теорема 6 [11]. С точностью до эквивалентности деформация антипуассоновской супералгебры с четным параметром $\hbar$ имеет вид

$$
\begin{aligned}
{[f(z), g(z)]_{*}=[} & f(z), g(z)]+ \\
& +(-1)^{\varepsilon(f)}\left\{\frac{c}{1+c N_{z} / 2} \Delta f(z)\right\} \varepsilon_{z} g(z)+\left\{\varepsilon_{z} f(z)\right\} \frac{c}{1+c N_{z} / 2} \Delta g(z),
\end{aligned}
$$

где

$$
N_{z}=\sum_{A} z_{A} \frac{\partial}{\partial z_{A}}, \quad \Delta=\sum_{i} \frac{\partial}{\partial x_{i}} \frac{\partial}{\partial \xi_{i}}
$$

$u c \in \hbar^{2} \mathbb{K}\left[\left[\hbar^{2}\right]\right]$.

\section{1. Центральное расширение антипуассоновской супералгебры $D_{n}$.}

Теорема 7 [20]. Имеют место следующие утверждения.

1. $H_{\mathrm{tr}}^{1} \simeq 0$.

2. Eсли $n \geqslant 2$, mo $H_{\mathrm{tr}}^{2} \simeq 0$. Eсли $n=1$, mо $H_{\mathrm{tr}}^{2} \simeq \mathbb{K}^{2}$ и кочепи

$$
\mu_{1}(f, g)=\int d z(-1)^{\varepsilon(f)}\left\{\partial_{x}^{3} \partial_{\xi} f(z)\right\} g(z), \quad \mu_{2}(f, g)=\int d z(-1)^{\varepsilon(f)}\left\{\partial_{x}^{2} f(z)\right\} g(z)
$$

являются независимыми нетривиальными коциклами. 
Таким образом, антипуассоновская супералгебра $D_{1}$ имеет центральные расширения.

Теорема 8 [11]. Централъное расширение антипуассоновской супералгебръ $D_{1}$ не имеет деформаций с четным параметром $\hbar$.

Благодарности. Настоящая работа поддержана РФФИ (гранты № 05-01-00996 (И. Тютин), № 05-02-17217 (С. Конштейн)) и Программой поддержки ведущих научных школ (грант № НШ-4401.2006.2).

\section{Список литературы}

[1] F. Bayen, M. Flato, C. Fronsdal, A. Lichnerovich, D. Sternheimer, Ann. Phys., 111:1 (1978), 61-110; Ann. Phys., 111:1 (1978), 111-151.

[2] М. В. Карасев, В.П. Маслов, Нелинейные скобки Пуассона. Геометрия и квантование, Наука, M., 1991.

[3] B. Fedosov, Deformation Quantization and Index Theory, Akademie, Berlin, 1996.

[4] M. Kontsevich, Lett. Math. Phys., 66:3 (2003), 157-216; arXiv: q-alg/9709040.

[5] Д. А. Лейтес, И. М. Щепочкина, ТМФ, 126:3 (2001), 339-369.

[6] И. В. Тютин, ТМФ, 128:3 (2001), 515-539.

[7] В. В. Жаринов, ТМФ, 136:2 (2003), 179-196.

[8] С. Е. Конштейн, А.Г. Смирнов, И.В. Тютин, ТМФ, 148:2 (2006), 163-178; arXiv: hep-th/0401023.

[9] S. E. Konstein, I. V. Tyutin, General form of the deformation of Poisson superbracket on $(2, n)$-dimensional superspace, arXiv: hep-th/0610308, предст. в ТМФ.

[10] S.E. Konstein, I. V. Tyutin, Deformations of the central extension of the Poisson superalgebra, arXiv: hep-th/0501027, предст. в ТМФ.

[11] S. E. Konstein, I. V. Tyutin, Deformations of the antibracket, arXiv: hep-th/0512313.

[12] M. Gerstenhaber, Ann. Math., 79:1 (1964), 59-103; 99:2 (1974), 257-276.

[13] M. Scheunert, R. B. Zhang, J. Math. Phys., 39:9 (1998), 5024-5061; arXiv: q-alg/9701037.

[14] С. Е. Конштейн, А.Г. Смирнов, И.В. Тютин, ТМФ, 143:2 (2005), 163-194; arXiv: hep-th/0312109.

[15] I. A. Batalin, G. A. Vilkovisky, Phys. Lett. B, 120:1-3 (1983), 166-170.

[16] I. A. Batalin, G. A. Vilkovisky, J. Math. Phys., 26:1 (1985), 172-184.

[17] J. Gomis, J. Paris, S. Samuel, Phys. Rep., 259:1-2 (1995), 1-145; arXiv: hep-th/9412228.

[18] Д. М. Гитман, И. В. Тютин, Каноническое квантование полей со связями, Наука, М., 1986.

[19] M. Henneaux, C. Teitelboim, Quantization of Gauge Systems, University Press, Princeton, NJ, 1992.

[20] S.E. Konstein, I. V. Tyutin, Cohomology of antiPoisson superalgebra, arXiv: hep-th/0512300. 